\title{
DETECÇÃO DE IMUNOGLOBULINAS ANTI-Toxoplasma gondii E ANTI-Neospora caninum EM BOVINOS NO RIO GRANDE DO SUL, BRASIL
}

\author{
João Luis Revolta Callefe ${ }^{1}$ \\ Helio Langoni ${ }^{2}$ \\ Karine Bott Mantovan ${ }^{3}$
}

\begin{abstract}
RESUMO
Neosporose e toxoplasmose são doenças de importância na esfera reprodutiva e em saúde pública. Dados de soroprevalência destes protozoários em regiões de importante cadeia produtiva de bovinos no Brasil, como a região Sul, são necessários para compreender a extensão dos problemas causados por estas enfermidades, bem como melhorar o emprego de medidas profiláticas e de controle. Os resultados do levantamento sorológico realizado por este estudo, pela reação de Imunofluorescência Indireta (IFI) para detecção de anticorpos antiT. gondii e anti- N. caninum, apontaram animais positivos para infecção por T. gondii $(7,2 \%)$ e $N$. caninum (7,6\%), abaixo das citadas na literatura no Estado do Rio Grande do Sul, que parece apresentar prevalências menores quando comparado com outras regiões do Brasil, caracterizando uma disseminação moderada destes Patógenos.
\end{abstract}

Palavras-chave: toxoplasmose bovina, neosporose bovina, bovinos, Rio Grande do Sul

\section{DETECTION OF ANTI- T. gondii AND ANTI- N. caninum IMMUNOGLOBULINS IN CATTLE IN THE STATE OF RIO GRANDE DO SUL}

\begin{abstract}
Neosporosis and toxoplasmosis are diseases of importance in the reproductive sphere and public health. Seroprevalence data for these protozoa in regions with major bovine productive chains in Brazil, such as the South, are necessary to understand the extent of the problems caused by these diseases, as well as to improve the use of prophylactic and control measures. The results of the serological survey carried out by this study, using the Indirect Immunofluorescence (IFI) reaction to detect antibodies against $T$. gondii and anti- $N$. caninum, showed positive animals for infection by $T$. gondii $(7.2 \%)$ and $N$. caninum $(7,6 \%)$, below those mentioned in the literature in the State of Rio Grande do Sul, which seems to have lower prevalences when compared to other regions of Brazil, characterizing moderate dissemination of these pathogens.
\end{abstract}

Keywords: bovine toxoplasmosis, bovine neosporosis, cattle, Rio Grande do Sul

\footnotetext{
${ }^{1}$ Universidade de São Paulo. joaocallefe@usp.br

${ }^{2}$ Faculdade de Medicina Veterinária e Zootecnia da Universidade Estadual Paulista Júlio de Mesquita Filho.

Correspondência: helio.langoni@unesp.br

${ }^{3}$ Universidade Estadual Paulista Julio de Mesquita Filho. karinebott@gmail.com
} 


\title{
DETECCIÓN DE INMUNOGLOBULINAS ANTI-Toxoplasma gondii Y ANTI- Neospora caninum EN GANADO EN RIO GRANDE DO SUL, BRASIL
}

\author{
RESUMEN
}

La neosporosis y la toxoplasmosis son enfermedades de importancia en el ámbito reproductivo y en la salud pública. Los datos de seroprevalencia de estos protozoos en regiones con una importante cadena productiva bovina en Brasil, como el Sur, son necesarios para comprender la magnitud de los problemas causados por estas enfermedades, así como para mejorar el uso de medidas profilácticas y de control. Los resultados de la encuesta serológica realizada por este estudio, utilizando la reacción de inmunofluorescencia indirecta (IFI) para detectar anticuerpos contra $T$. gondii y anti- $N$. caninum, mostraron animales positivos para infección por $T$. gondii $(7,2 \%)$ y $N$. caninum $(7,6 \%)$, por debajo de los mencionados en la literatura en el estado de Rio Grande do Sul, que parece tener prevalencias menores en comparación con otras regiones de Brasil, caracterizando una diseminación moderada de estos agentes.

Palabras clave: toxoplasmosis, neosporosis, ganado, Rio Grande do Sul

\section{INTRODUÇÃO}

Os bovinos apresentam grande importância no ciclo biológico de enfermidades parasitárias como neosporose e toxoplasmose, atuando como hospedeiros intermediários em ambas protozooses. Infecções por Neospora caninum podem gerar prejuízos econômicos de caráter reprodutivo para produtores de gado, quanto que bovinos infectados com Toxoplasma gondii podem ser fonte de infecção do agente ao ser humano, criando grande preocupação no âmbito de saúde pública.

Em relação a clínica em bovinos, alguns autores apontam que não há relação significante entre sorologia positiva para $T$. gondii e problemas reprodutivos como morte fetal a abortamento (1). De maneira geral, a prevalência do $T$. gondii em rebanhos destinados a consumo humano, pode variar de $19,2 \%$ a $71 \%(2,3)$, o que reforça a importância do parasita nos bovídeos, já que eles são uma das principais rotas de transmissão para humanos a partir do consumo de carne mal cozida ou crua contendo cistos do parasita (4). No Rio Grande do Sul, de acordo com o estudo de F. Santos et al. (5) a prevalência resultante foi de $17,4 \% \mathrm{em}$ bovinos destinados a produção de carne.

O diagnóstico pode ser realizado de forma direta, evidenciando a presença do parasita por exame parasitológico por meio do isolamento in vivo ou in vitro, métodos moleculares ou, ou indiretamente, pela detecção de anticorpos específicos contra $T$. gondii por métodos sorológicos como a reação de Imunofluorescência Indireta (IFI). Apesar da facilidade de diagnóstico por estas técnicas, é preciso ter cautela em analisar os resultados, pois não há diferenciação entre animais doentes e infectados, devendo-se então associar a variação de títulos encontrados ao estado clínico do animal. Bem como a toxoplasmose, a neosporose também acomete os animais domésticos, e tem como agente etiológico o Neospora caninum. No tecido de bovinos, seus hospedeiros intermediários, são observadas as formas de taquizoitos e bradizoitos. Normalmente, em bovinos adultos, o protozoário persiste no organismo na forma de bradizoitos encistados.

Para complementar o papel do gado no ciclo epidemiológico da neosporose, acredita-se que os resíduos placentários infectados com $N$. caninum, apresentam grande importância na infecção de cães e outros canídeos. Esta ideia pode ser apoiada pelo estudo de Fioretti et al. (6) que isolaram o parasita de restos placentários de vacas naturalmente infectadas, que 
produziram bezerros infectados.

A transmissão da neosporose no gado é considerada bastante eficiente de modo que possui rotas de transmissões horizontal (transmissão placentária exógena) e vertical (transmissão placentária endógena) (7), contribuindo para a manutenção da doença no plantel para gerações futuras (8), além de poder gerar abortamento, na maioria das vezes, em animais saudáveis (9). $N$. caninum pode ser excretado por secreções como o leite e descargas uterinas de vacas infectadas, o que pode contribuir para a contaminação de produtos, bem como de outros animais em uma propriedade, devida a eliminação de taquizoítos (10).

O diagnóstico da neosporose pode ser feito de diferentes maneiras, devendo-se levar em conta fatores como a clínica do animal, histórico da propriedade e exames complementares. Os testes sorológicos são de grande importância e utilidade; diferentes técnicas sorológicas estão disponíveis e são baseadas na detecção de anticorpos séricos contra a forma de taquizoítos, por exemplo, a reação de Imunofluorescência Indireta (IFI) e teste imunoenzimático (ELISA) (11).

A importância da toxoplasmose e neosporose é evidente, uma vez que ambas podem afetar a cadeia produtiva, contribuindo para condenação ou desvalorização de carcaças, gerando problemas na esfera reprodutiva e afetando a saúde pública. Por isso, é importante que estudos sorológicos sejam realizados, principalmente nas regiões que representam grande parte da população do gado brasileiro.

\section{MATERIAL E MÉTODOS}

As 265 amostras de soro bovino utilizadas no presente estudo foram obtidas por conveniência a partir de um banco de amostras do Serviço de Diagnóstico de Zoonoses (SDZ) da Faculdade de Medicina Veterinária e Zootecnia da Universidade Estadual Paulista "Júlio de Mesquita Filho" (Botucatu-SP), para inquérito sorológico de diferentes enfermidades, provenientes de 19 municípios do estado do Rio Grande do Sul. O soro de cada bovino passou por uma triagem sorológica para detecção de imunoglobulinas anti- $T$. gondii e anti- $N$. caninum, mediante a diluição titulação de 1:16 e 1:25 (11), respectivamente, utilizando-se a técnica de Reação de Imunofluorescência Indireta. As amostras reagentes na triagem para $T$. gondii, foram submetidas ao mesmo protocolo de triagem de Camargo (12), porém aplicandose as diluições de 1:16, 1:64, 1:256 e assim sucessivamente até que os títulos sorológicos se extinguissem. Para detecção de anticorpos anti- N. caninum, as amostras reagentes na diluição de 1:25, foram submetidas a titulação de 1:25, 1:50, 1:100, 1:200 e 1:400.

\section{RESULTADOS E DISCUSSÃO}

Analisando as regiões com grande representatividade do gado bovino brasileiro, a região sul do país fica em destaque, composta pelos estados do Paraná, Rio Grande do Sul e Santa Catarina e, de modo geral, tem sido um dos maiores responsáveis pela produção brasileira de leite (13).

Dentre as 265 amostras triadas, provenientes de dezenove municípios do estado do Rio Grande do Sul, para a detecção de anticorpos anti- T. gondii, 33 (12,5\%) foram consideradas reagentes para que, a seguir, fossem tituladas de acordo com Camargo (12). Catorze amostras apresentaram título 16 UI, 10 de 64 UI, 7 de 256 UI, 1 com título 1024 UI e 1 título de 4096 UI. Segundo a recomendação de Santos et al. (14), foram consideradas positivas as amostras com titulação a partir de 64 UI, portanto, 19 das 265 amostras de soro bovino apresentaram resultados positivos, conforme a Tabela 1. Deste modo, apenas 10 das 19 localidades avaliadas, apresentaram amostras positivas: Alecrim, Alegrete, Candiota, Dom Pedrito, Herval, Jaguarão, Pedras Altas, Nova Roma do Sul, Seberi e Uruguaiana. 


\begin{tabular}{|c|c|c|c|c|c|c|c|c|}
\hline Anti- T. gondii & Município & Amostras totais & $\begin{array}{c}\text { Amostras triadas para } \\
\text { titulação }(>0 u=1: 16)\end{array}$ & $1: 16$ & $1: 64$ & $1: 256$ & $1: 1024$ & $1: 4096$ \\
\hline & Alecrim & 15 & 2 & 0 & 0 & 2 & 0 & 0 \\
\hline & Alegrete & 15 & 2 & 0 & 0 & 2 & 0 & 0 \\
\hline & Bajé & 15 & 0 & 0 & 0 & 0 & 0 & 0 \\
\hline & Candiota & 11 & 3 & 0 & 1 & 2 & 0 & 0 \\
\hline & Coqueiros do Sul & 13 & 0 & 0 & 0 & 0 & 0 & 0 \\
\hline & Dom Pedrito & 15 & 2 & 0 & 1 & 0 & 1 & 0 \\
\hline & Herval & 14 & 4 & 0 & 4 & 0 & 0 & 0 \\
\hline & Itaqui & 15 & 0 & 0 & 0 & 0 & 0 & 0 \\
\hline & Jaguarão & 15 & 2 & 0 & 1 & 0 & 0 & 1 \\
\hline & \begin{tabular}{|l|} 
Nova Roma do Sul \\
\end{tabular} & 15 & 6 & 5 & 1 & 0 & 0 & 0 \\
\hline & Pedras Altas & 11 & 1 & 0 & 0 & 1 & 0 & 0 \\
\hline & Piratini & 15 & 0 & 0 & 0 & 0 & 0 & 0 \\
\hline & \begin{tabular}{|l|} 
Porto Alegre \\
\end{tabular} & 15 & 2 & 2 & 0 & 0 & 0 & 0 \\
\hline & \begin{tabular}{|l|} 
Rosário do Sul \\
\end{tabular} & 10 & 0 & 0 & 0 & 0 & 0 & 0 \\
\hline & Seberi & 15 & 5 & 4 & 1 & 0 & 0 & 0 \\
\hline & Santa Vitória do Palmar & 15 & 0 & 0 & 0 & 0 & 0 & 0 \\
\hline & São Borja & 13 & 0 & 0 & 0 & 0 & 0 & 0 \\
\hline & \begin{tabular}{|l|} 
Tenente Portela \\
\end{tabular} & 13 & 0 & 0 & 0 & 0 & 0 & 0 \\
\hline & Uruguaiana & 13 & 4 & 3 & 1 & 0 & 0 & 0 \\
\hline TOTAI. & & 265 & 33 & 14 & 10 & 7 & 1 & 1 \\
\hline Anti- $N$. caninum & Município & Amostras totais & $\begin{array}{l}\text { Amostras triadas para } \\
\text { titulação }(>0 u=1: 25)\end{array}$ & $1: 25$ & $1: 50$ & $1: 100$ & $1: 200$ & $1: 400$ \\
\hline & Alecrim & 15 & 5 & 4 & 1 & 0 & 0 & 0 \\
\hline & Alegrete & 15 & 2 & 2 & 0 & 0 & 0 & 0 \\
\hline & Bajé & 15 & 4 & 3 & 1 & 0 & 0 & 0 \\
\hline & Candiota & 11 & 2 & 1 & 0 & 0 & 0 & 1 \\
\hline & \begin{tabular}{|l|} 
Coqueiros do Sul \\
\end{tabular} & 13 & 4 & 2 & 2 & 0 & 0 & 0 \\
\hline & Dom Pedrito & 15 & 6 & 4 & 1 & 0 & 0 & 1 \\
\hline & Herval & 14 & 0 & 0 & 0 & 0 & 0 & 0 \\
\hline & Itaqui & 15 & 4 & 2 & 1 & 1 & 0 & 0 \\
\hline & Jaguarão & 15 & 6 & 1 & 2 & 1 & 0 & 2 \\
\hline & Nova Roma do Sul & 15 & 5 & 0 & 2 & 1 & 1 & 1 \\
\hline & Pedras Altas & 11 & 6 & 3 & 1 & 2 & 0 & 0 \\
\hline & Piratini & 15 & 7 & 3 & 1 & 2 & 1 & 0 \\
\hline & Porto Alegre & 15 & 1 & 0 & 0 & 0 & 0 & 1 \\
\hline & Rosário do Sul & 10 & 1 & 1 & 0 & 0 & 0 & 0 \\
\hline & Seberi & 15 & 3 & 2 & 0 & 1 & 0 & 0 \\
\hline & Santa Vitória do Palmar & 15 & 2 & 0 & 1 & 0 & 1 & 0 \\
\hline & São Borja & 13 & 1 & 0 & 0 & 0 & 1 & 0 \\
\hline & Tenente Portela & 13 & 5 & 2 & 1 & 0 & 0 & 2 \\
\hline & Uruguaiana & 13 & 2 & 1 & 1 & 0 & 0 & 0 \\
\hline TOTAL & & 265 & 66 & 31 & 15 & 8 & 4 & 8 \\
\hline
\end{tabular}

Tabela 1: Distribuição entre sorologias para T. gondii e $N$. caninum em 19 municípios do estado do Rio Grande do Sul

Analisando os resultados obtidos para detecção de anticorpos anti- N. caninum, 66 $(24,9 \%)$ amostras foram reagentes e submetidas a titulação por apresentarem fluorescência na triagem, correspondente a diluição de 1:25. Dentre as 265 amostras, 31 apresentaram título de 25 UI, 15 com título de 50 UI, 8 de 100 UI, 4 de 200 UI e, por fim, 8 amostras apresentaram título de 400 UI. Doze dos 19 municípios, apresentaram amostras positivas dentre os 66 soros titulados, conforme a Tabela 1: Candiota, Dom Pedrito, Itaqui, Jaguarão, Nova Roma do Sul, Pedras Altas, Piratini, Porto Alegre, Saberi, Santa Vitoria do Palmar, São Borja e Tenente Portela. De acordo com Boas et al. (15) e Aguiar et al. (16), títulos maiores ou iguais a 100 UI são considerados como ponto de corte para infecção por $N$. caninum. Dentre as amostras analisadas, apenas 20 foram consideradas positivas e sugestivas de infecção para $N$. caninum, representando 7,6\% de animais infectados.

Outros estudos realizados no Rio Grande do Sul sugerem uma ocorrência maior do que a encontrada pelo presente trabalho, tanto para detecção de anticorpos anti- N. caninum, como para anti- T. gondii. Foram considerados positivos para infecção por T. gondii os animais com títulos maiores ou iguais a 1:64. Suaréz-Aranda et al. (2) e Santos et al. (3) relatam prevalências de anticorpos anti- T. gondii que variam de $19,2 \%$ a $71 \%$ nos rebanhos bovinos, e Santos et al. (5) obtiveram a prevalência de 17,4\% em bovinos destinados a produção de carne.

Para N. caninum, foi demonstrado por Lucas (17) uma prevalência de 12,04\% em estudo realizado em quatro municípios do estado do Rio Grande do Sul. Outro estudo, 
também realizado no Rio Grande do Sul em vacas de leite, revelou a soroprevalência de $11,2 \%$, demostrando inclusive associação com abortamentos ocorridos na região (18). A soroprevalência em outras regiões do Brasil parece ser mais elevada de acordo com Santos et al. (3), Ogawa et al. (19), Melo et al. (20) e Teixeira et al. (21).

\section{CONCLUSÃO}

A neosporose em bovinos é uma enfermidade de grande importância para produção por colocar estes animais em risco de complicações reprodutivas e, consequentemente, ocasionar perdas econômicas para pecuaristas. Quando tratamos de toxoplasmose, há grande preocupação quanto aos impactos negativos a saúde pública. Para mitiga-los, é imprescindível entender como a distribuição da doença ocorre em determinada região e, por isso, o acompanhamento sorológico é fundamental. O presente estudo obteve ocorrência de resultados positivos para infecção por $T$. gondii $(7,2 \%)$ e $N$. caninum $(7,6 \%)$, abaixo das citadas na literatura no estado do Rio Grande do Sul.

\section{REFERÊNCIAS}

1. Canada N, Meireles CS, Rocha A, Correia da Costa JM, Erickson MW, Dubey JP. Isolation of viable Toxoplasma gondii from naturally infected aborted bovine fetuses [Internet]. J Parasitol [Internet]. 2002 [cited 2019 Aug 4];88(6):1247-8. Available from: https://naldc.nal.usda.gov/download/49040/PDF

2. Suaréz-Aranda F, Galisteo AJ, Hiramoto RM, Cardoso RP, Meireles LR, Miguel O, et al. The prevalence and avidity of Toxoplasma gondii IgG antibodies in pigs from Brazil and Peru. Vet Parasitol [Internet]. 2000 [cited 2019 Aug 4];91(1-2):23-32. Available from: http://www.ncbi.nlm.nih.gov/pubmed/10889357

3. Santos TR, Costa AJ, Toniollo GH, Luvizotto MCR, Benetti AH, Santos RR, et al. Prevalence of anti-Toxoplasma gondii antibodies in dairy cattle, dogs, and humans from the Jauru micro-region, Mato Grosso state, Brazil. Vet Parasitol [Internet]. 2009 [cited 2019 Aug 4];161(3-4):324-6. Available from: http://www.ncbi.nlm.nih.gov/pubmed/19232473

4. Dubey JP. Strategies to reduce transmission of Toxoplasma gondii to animals and humans. Vet Parasitol [Internet]. 1996 [cited 2019 Aug 4];64(1-2):65-70. Available from: http://www.ncbi.nlm.nih.gov/pubmed/8893464

5. Santos LF, Damé M, Cademartori B, Cunha Filho N, Farias NR, Ruas J. Occurrence of antibodies to Toxoplasma gondii in water buffaloes and meat cattle in Rio Grande do Sul State, southern Brazil. Acta Parasitol [Internet]. 2013 [cited 2019 Aug 4];58(3):334-6. Available from: http://www.degruyter.com/view/j/ap.2013.58.issue-3/s11686-013-01484/s11686-013-0148-4.xml

6. Fioretti DP, Pasquali P, Diaferia M, Mangili V, Rosignoli L. Neospora caninum infection and congenital transmission: serological and parasitological study of cows up to the fourth gestation. J Vet Med B Infect Dis Vet Public Health [Internet]. 2003 [cited 2019 Aug 4];50(8):399-404. Available from: http://www.ncbi.nlm.nih.gov/pubmed/14633211 
7. Dubey JP, Buxton D, Wouda W. Pathogenesis of bovine neosporosis. J Comp Pathol [Internet]. 2006 [cited 2019 Aug 4];134(4):267-89. Available from: https://linkinghub.elsevier.com/retrieve/pii/S0021997505001350

8. Wouda W, Moen AR, Schukken YH. Abortion risk in progeny of cows after a neospora caninum epidemic. Theriogenology [Internet]. 1998 [cited 2019 Aug 4];49(7):1311-6. Available from: http://www.ncbi.nlm.nih.gov/pubmed/10732068

9. Anderson ML, Reynolds JP, Rowe JD, Sverlow KW, Packham AE, Barr BC, et al. Evidence of vertical transmission of Neospora sp. infection in dairy cattle. J Am Vet Med Assoc [Internet]. 1997 [cited 2019 Aug 4];210(8):1169-72. Available from: http://www.ncbi.nlm.nih.gov/pubmed/9108925

10. Davidson H, Guy C, McGarry J, Guy F, Williams DJ, Kelly D, et al. Experimental studies on the transmission of Neospora caninum between cattle. Res Vet Sci [Internet]. 2001 [cited 2019 Aug 4];70(2):163-8. Available from: http://www.ncbi.nlm.nih.gov/pubmed/11356096

11. Andreotti R, Locatelli-Dittrich R, Soccol VT, Paiva F. Diagnóstico e controle da neosporose em bovinos [Internet]. Campo Grande: Embrapa Gado de Corte; 2003 [cited 2019 Aug 4]. Available from: https://www.embrapa.br/gado-de-corte/busca-depublicacoes/-/publicacao/324070/diagnostico-e-controle-da-neosporose-em-bovinos

12. Camargo, ME. Introdução às técnicas de imunofluorescência. Rev Bras Patol Clin. 1974;10(3):143-71.

13. Zoccal R, Pereira VF. Panorama do leite [Internet]. Juiz de Fora (MG): Embrapa Gado de Leite; 2016 [cited 2019 Aug 4]. Available from: https://ainfo.cnptia.embrapa.br/digital/bitstream/item/156066/1/Cnpgl-2016-

PanoramadoLeite-v8-n88-jun.pdf

14. Santos APME, Navarro IT, Bracarense APFRL, Freire RL, Marana ERM, Ogawa L, et al. Dairy cow abortion associated with Neospora caninum and other infectious agents. Arq Bras Med Vet Zootec [Internet]. 2005 [cited 2019 Aug 4];57(4):545-7. Available from: http://www.scielo.br/scielo.php?script=sci_arttext\&pid=S010209352005000400017\&lng=en\&tlng=en

15. Boas RV, Pacheco TA, Melo ALT, Oliveira ACS, Aguiar DM, Pacheco RC. Infection by Neospora caninum in dairy cattle belonging to family farmers in the northern region of Brazil. Rev Bras Parasitol Vet. 2015;24(2):204-8. doi: https://doi.org/10.1590/S198429612015035.

16. Aguiar DM, Lacerda DP, Orlandelli RC, Medina AO, Azevedo SS, Okuda LH, et al. Seroprevalence and risk factors associated to Neospora caninum in female bovines from the western São Paulo State, Brazil. Arq Inst Biol. 2011;78(2):183-9. doi: https://doi.org/10.1590/1808-1657v78p1832011.

17. Lucas AS. Neospora caninum em bovinos da bacia leiteira da região de Pelotas, RS: soroprevalência e associação com fatores de risco [Internet]. Pelotas: Universidade 
Federal de Pelotas; 2007 [cited 2019 Aug 4]. Available from: http://repositorio.ufpel.edu.br/handle/ri/2524

18. Corbellini LG, Driemeier D, Cruz CFE, Gondim LFP, Wald V. Neosporosis as a cause of abortion in dairy cattle in Rio Grande do Sul, southern Brazil. Vet Parasitol [Internet]. 2002 [cited 2019 Aug 4];103(3):195-202. Available from: http://www.ncbi.nlm.nih.gov/pubmed/11750112

19. Ogawa L, Freire RL, Vidotto O, Gondim LFP, Navarro IT. Occurrence of antibodies to Neospora caninum and Toxoplasma gondii in dairy cattle from the northern region of the Paraná State, Brazil. Arq Bras Med Vet Zootec [Internet]. 2005 [cited 2019 Aug 4];57(3):312-6. Available

from: http://www.scielo.br/scielo.php?script=sci_arttext\&pid=S010209352005000300006\&lng=en\&tlng=en

20. Melo DPG, Silva AC, Ortega-Mora LM, Boaventura CM. Prevalência de anticorpos Anti-Neospora caninum em bovinos das microrregiões de Goiânia e Anápolis, Goiás, Brasil. Rev Bras Parasitol Vet [Internet]. 2006 [cited 2019 Aug 4];15(3):105-9. Available from: http://www.redalyc.org/articulo.oa?id=397841459004

21. Teixeira WC, Uzêda RS, Gondim LFP, Silva MIS, Pereira HM, Alves LC, et al. Prevalência de anticorpos anti-Neospora caninum (Apicomplexa: Sarcocystidae) em bovinos leiteiros de propriedades rurais em três microrregiões no estado do Maranhão. Pesqui Vet Bras [Internet]. 2010 [cited 2019 Aug 4];30(9):729-34. Available from: http://www.scielo.br/scielo.php?script=sci_arttext\&pid=S0100-

736X2010000900004\&lng=pt\&tlng=pt

Recebido em: 16/11/2020 Aceito em: 09/03/2021 